\title{
2 dof robust controller design using fopdt model
}

\author{
Mohammed Shoeb Mohiuddin, Anuj Kumar, Suraj Kumhar \\ Assistant Professor, Department of Electrical Engineering \\ Associate lecture, Department of Electrical Engineering \\ Mewar University, Chittorgarh, Rajasthan, India
}

\begin{abstract}
The main goal of a control system is that of causing a dynamic process to behave in a desired manner. The analysis and design of such a control system to provide a demanded behaviour is usually done by employing a mathematical model of the dynamic process. This model is chosen to represent the major dynamical features of the process. For the reason that the mathematical model is an idealization of the real process, it is imprecise and this inaccuracy entails the existence of model uncertainty. This fact, among others, complicates the analysis and design of a control system. The choice of the control structure plays an essential role to allow the attainment of the demanded behaviour. Typically, some kind of specifications, for example, open-loop and closed-loop specifications, cannot be fulfilled simultaneously and trade off between them has to be considered.

Therefore, it is important to distinguish between difficulties to the control problem (such as model uncertainty, disturbances that cause the output to deviate from its desired value, etc.) and difficulties due to the control structure. In this way, the work has been focused on attempting to find out and use new control structures to avoid traditional difficulties related with standard, well-established, feedback control configurations.

Thus in this thesis, a new 2-DOF control configuration based on plant processes which are first approximated to First-Order plus dead time (FOPDT) models, then the reference and feedback controllers are tuned according to the plant parameters itself.
\end{abstract}

Keywords: dof:fopdt

\section{Introduction}

A primary objective of a control system is to make the output of a dynamic process behave in a certain manner. This desired behaviour for the output is pursued by means of manipulations on the input of the process. Nevertheless, hard constraints such as limits on the controls or states and performance objectives prevents the accomplishment of the desired behaviour for the controlled process. The design procedure of a control system usually involves a mathematical model of the dynamic process, the plant model or nominal model. Consequently, many aspects of the real plant behaviour cannot be captured in an accurate way with the plant model leading to uncertainties. Such plant model mismatching should be characterized, albeit loosely, by means of disturbances signals and/or plant parameter variations, often characterized by probabilistic models, or unmodelled dynamics, commonly characterized in the frequency domain. Usually, high performance specifications are given in terms of the plant model. For this reason, model uncertainties characterization should be incorporated to the design procedure in order to provide a reliable control system capable to deal with the real process and to assure the fulfillment of the performance requirements. The term robustness is used to denote the ability of a control system to cope with the uncertain scenario. The performance specifications are usually given accordingly for the regulation problem or for the tracking problem. The former is to manipulate the input of the plant to counteract the effect of output disturbances. The later is to handle the input of the plant to keep the controlled variables close to the given reference signal. The key point is the way the controller generates the control signal in a suitable manner. There exist a lot of different strategies and methodologies to cope with this problem, say the controller design problem. However, any possible choice can be classified as belonging to open-loop control or closed loop control. 


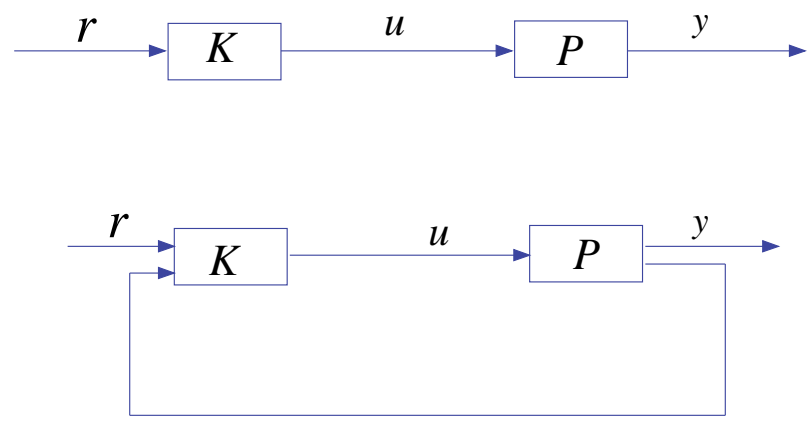

Figure 1.1 Open loop and closed loop control configurations

\subsection{Control system design requirements}

It is well known that there is an intrinsic conflict between performance and robustness in the standard feedback framework, discussed in $[1,2]$ for a detailed analysis and discussions. It is argued that the system response to commands is an open-loop property while robustness properties are associated with the feedback. Therefore, one must make a trade off between achievable performance (in terms command tracking) and robustness (against external disturbances and model uncertainties). In this way, a high performance controller designed for a nominal model may have very little robustness against the model uncertainties and the external disturbances.

For this reason, worst-case robust control design techniques such as $H_{\infty}$ control, one degree of freedom 1-DOF control, [3, 4], among many others have gained popularity in the last twenty years, or so. Unfortunately, it is well recognized in the robust control community that a robust controller design is usually achieved at the expense of performance.

\section{Proposed Algorithm}

In design procedure, First Order plus Dead Time (FOPDT) model is used. The aim is to have good set point tracking and disturbance rejection and also maximum robustness to model uncertainties. The tuning strategy is based on using analytical rules and some conceptual rules about closed loop poles and also an exhaustive search.

\section{First-Order plus dead time (FOPDT) process model:}

Deadtime $(\theta)$ is the time delay between the process and the sensor. Deadtime exists because no measurement or response to a process can be truly instantaneous. Although all systems have some degree of deadtime, too much deadtime can lead to problems with system response. If the deadtime is not appropriately accounted for, the lag in data readings can have detrimental effects on the implementation of control.

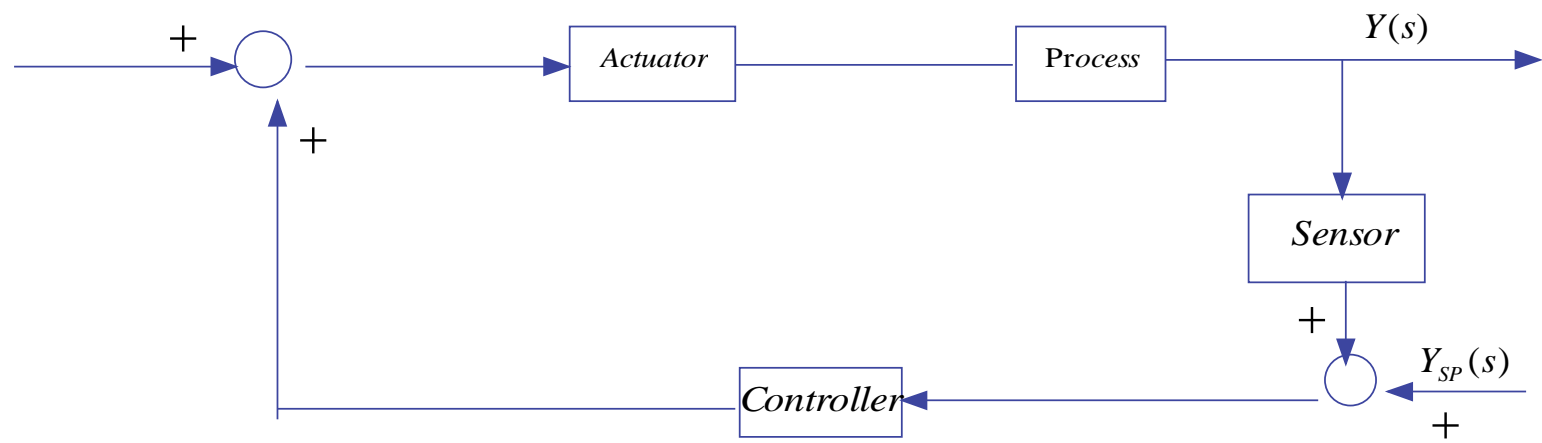

Figure Error! No text of specified style in document..1 First order plus dead time process model

The transfer function for deadtime is:

$G_{P}(s)=e^{-\theta_{p} s}$

\subsubsection{General dead time rule}

If $\theta<\tau_{P}$ the control system is not terribly hindered by deadtime 
If $\theta>\tau_{P}$ the control system is going to have problems accounting for deadtime and a model-based deadtime compensator should be used.

The first-order plus dead time (FOPDT) model can be used to approximate higher order processes. FOPDT models are better at approximating industrial processes than other dynamic models.

\section{FOPDT model}

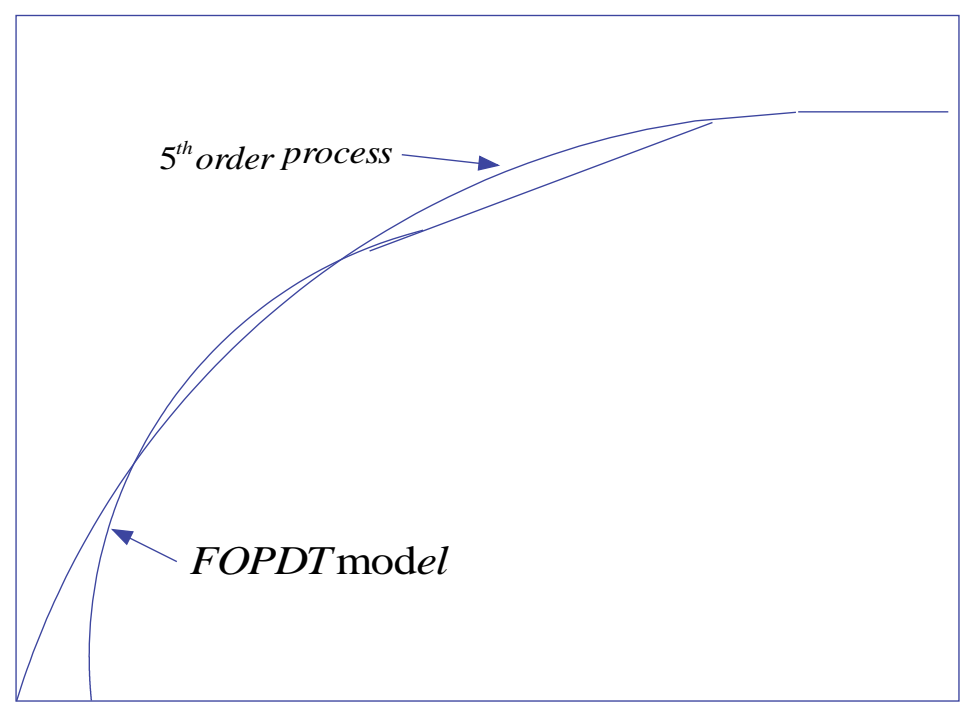

Time

Figure Error! No text of specified style in document..2 Comparison of $5^{\text {th }}$ order process and its FOPDT model

FOPDT models are the combination of a first-order process model with deadtime

$G_{p}(s)=\frac{K_{p} e^{-\theta_{p} s}}{\tau_{p} s+1}$

where $\tau_{P \text { and }} \theta_{P}$ are FOPDT parameters given by,

$\tau_{p}=\frac{t_{2 / 3}-t_{1 / 3}}{.7}$

$\theta_{p}=t_{1 / 3}-0.4 \tau_{p}$

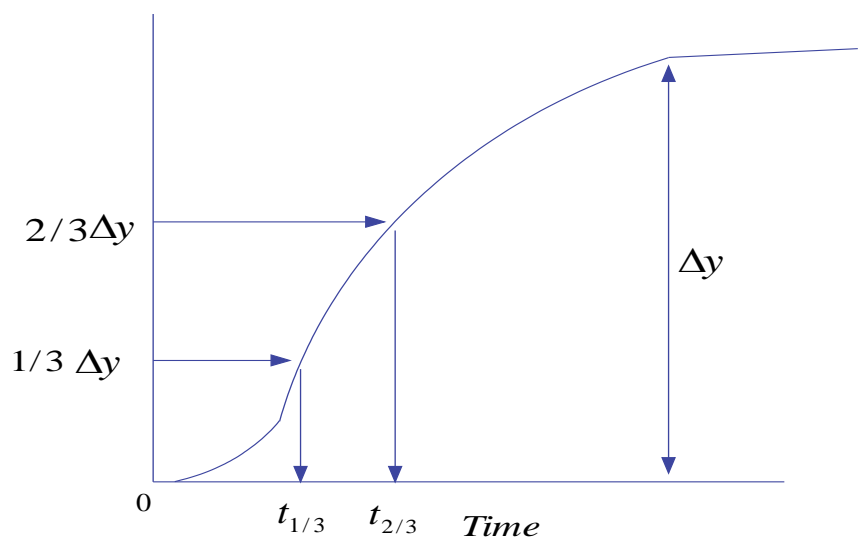

Figure Error! No text of specified style in document..3 Calculation of FOPDT parameters

\subsubsection{Padé Approximation}

This useful relationship allows for the approximation of the deadtime term in the FOPDT transfer function. The approximation represents the exponential function in terms of linear variables. 


$$
e^{-\theta_{p} s} \approx \frac{1-\frac{1}{2} \theta_{p} s}{1+\frac{1}{2} \theta_{p} s}
$$

of specified style in document..5)

(Error! No text

\subsubsection{How to interpret step-response data?}

When looking at a first-order response to a step change in the set point, there are three main variables. They are: Lag time $\left(\theta_{\mathrm{p}}\right.$, for a FOPDT system)

Process gain $\left(\mathrm{K}_{\mathrm{p}}\right)$

Process

time constant $\left(\tau_{\mathrm{p}}\right)$

These values can all be found by a graph of the system with a step increase as shown below:

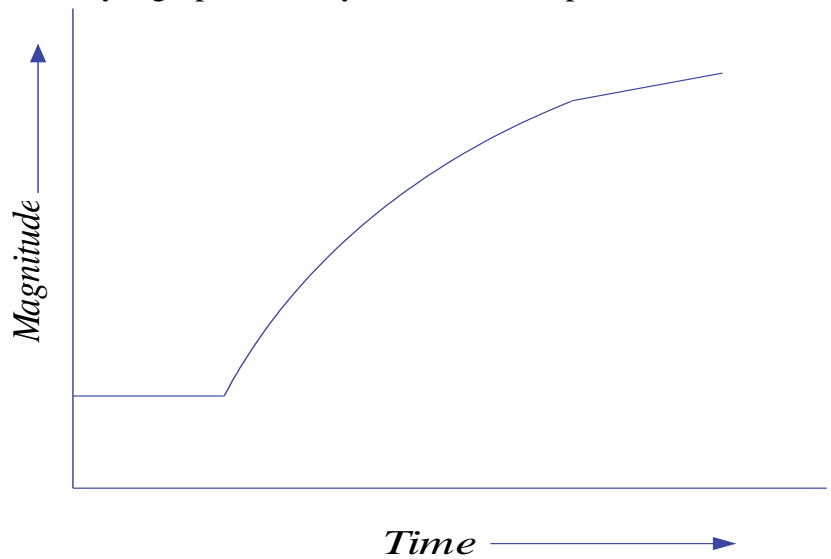

Figure Error! No text of specified style in document..4 Response of the system with step increase

If the slope approaches zero over time or reaches a steady-state value, then the system is considered to be stable. If the slope oscillates then the system is unstable. Instability prevents the determination of the process lag time, gain, and time constant.

All first order systems will display similar behaviour. The need to approximate dead time is characterized by a delay between $t=0$ and when the system begins to display first-order behaviour. In reality, nearly all processes will have some amount of deadtime. Finally the process lag time, gain, and time constant can be determined.

\subsubsection{Determining lag time}

1. Draw a line tangent to the response at the inflection point

2. Extend the line to the original set point (y-value). The $x$-value at the intersection of these values is the lag time (deadtime).

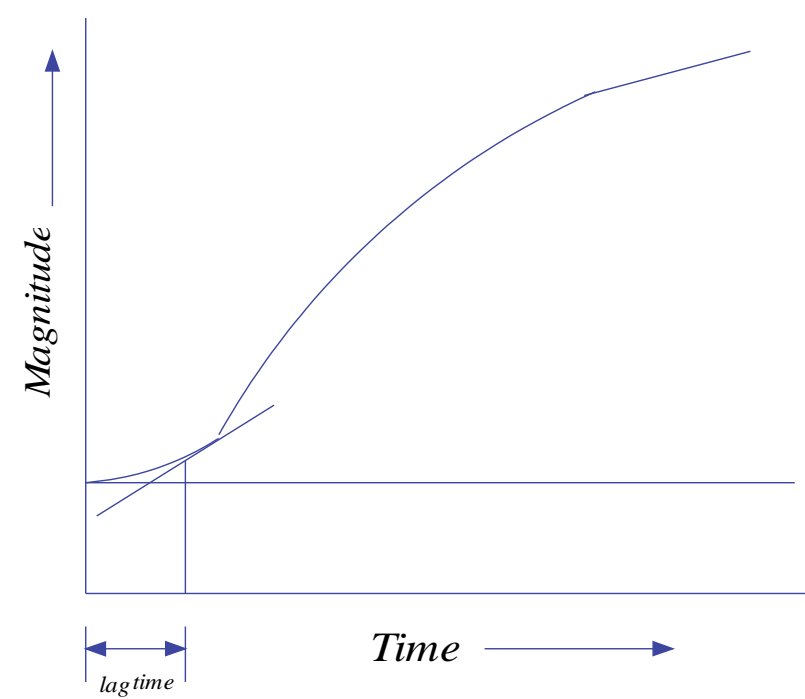

Figure Error! No text of specified style in document..5 Method to determine lag time 


\subsubsection{Determining process gain}

The process gain is defined by the change in process output divided by the magnitude of the set point change. This method can be used to calculate the process gain of both first order and FOPDT systems using the relation

$$
K_{p}=\frac{\Delta y}{\Delta u}
$$

of specified style in document..6)

\subsubsection{Determining the process time constant}

The process time constant $\left(\tau_{\mathrm{p}}\right)$ describes how long a system takes to approach a new steady-state value. The following methodology can be used to determine the $\tau_{\mathrm{p}}$ given a set of data.

(i) Use the first-order dynamics equation in the time domain to find specific ratios of $t$ to $\tau_{\mathrm{p}}$.

(ii) When $\mathrm{t}=\tau_{\mathrm{p}}, 63.2 \%$ of the total change in output has occurred. When $\mathrm{t}=2 * \tau_{\mathrm{p}}, 86.4 \%$ of the total change in output is reached, and when $\mathrm{t}=3 * \tau_{\mathrm{p}}, 95 \%$ of the total change has been obtained.

(iii) Multiply these percentages by the total change in output $(\Delta y)$ and add the initial output value. (in the picture below, $1+0.632 * 9=6.8,1+0.864 * 9=8.8$ etc $\ldots)$

(iv) Find the time on the $\mathrm{x}$-axis that corresponds to the output value calculated.

(v) Determine the average between any two of the time values determined to find the process time constant

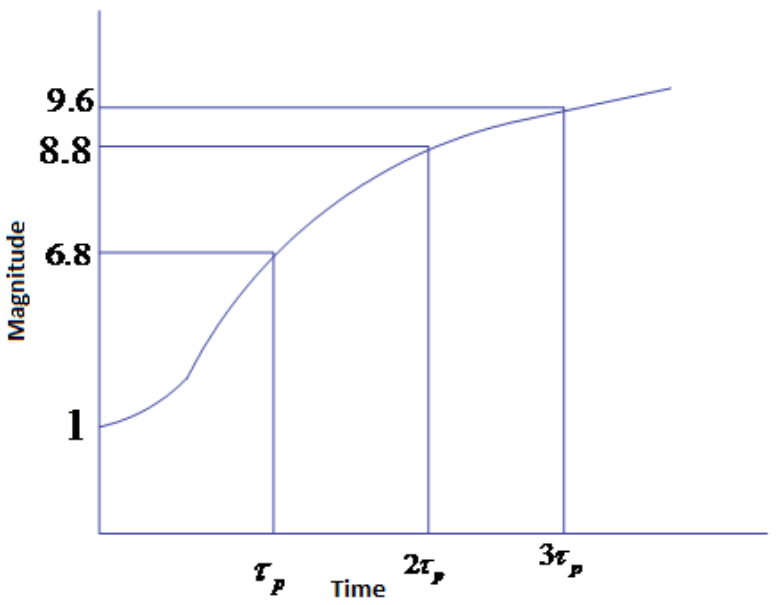

Figure Error! No text of specified style in document..6 Method to determine process time constant

\subsection{2-DOF PI controller structure}

The process is described by transfer function $G_{c}(s)$ and $G_{f f}(s)$ are respectively the feedback controller and feed forward controllers and are typical PI controllers.

$$
G_{c}(s)=k_{c}\left(1+\frac{1}{T_{i} s}\right)
$$

(Error! No

text of specified style in document..7)

$$
G_{f f}(s)=k_{c}\left(b+\frac{1}{T_{i} s}\right)
$$

(Error! No

\section{text of specified style in document..8)}

Parameter b in $G_{f f}(s)$ has no influence on disturbance rejection but it has a significant influence on the set point regulation. The tuning method is based on using First Order Plus Delay Time (FOPDT) model of process. It is a common approach to approximate processes by a FOPDT transfer function if it is possible. A large number of industrial processes can be approximately modelled by it. A FOPDT transfer function is given below

$G_{p}(s)=\frac{k_{p} e^{-\tau s}}{\tau s+1}$

(Error! No

text of specified style in document..9) 
where $k_{p}, \mathrm{~T}$ and $\tau$ are called process gain, time constant and dead time, respectively. A simple method to obtain FOPDT model of process is based on analysis of open loop step response for stable and minimum phase processes as described in equation (3.1).

\subsubsection{Control Requirements}

The design objective is to tune the controller parameters to achieve three main goals:

(i)Good disturbance rejection.

(ii)Good set point response.

(iii)Robustness to model uncertainties in FOPDT model.

Load disturbances in process control are low frequency signals added to control signal at the process input and drive the system away from its desired operating point. Good load disturbance rejection is achieved through minimizing the integrated absolute error (IAE) criterion for a step signal in disturbance input [41]

$$
I A E=\int_{0}^{\infty}|e(t)| d t
$$

of specified style in document..10)

(Error! No text

The second goal is to have a good set point tracking. These two goals are in conflict and good design for disturbance rejection may result bad set-point tracking. Using set point weighting in 2DOF structure solves this problem. In model based methods, the controller parameters typically are obtained from the model of process. Due to model uncertainties, the controller parameters should be tuned in a way that minimum sensitivity to these uncertainties is achieved. The robustness criterion is given as:

$$
\|S(j \omega)\|_{\infty}=\max \left|\frac{1}{1+G_{c}(j \omega) G_{p}(j \omega)}\right|
$$

specified style in document..11)

(Error! No text of

$\|S(j \omega)\|_{\infty}$ is called maximum value of the sensitivity function over entire frequency range, that is often used as a robustness measure since $\| s\left(j \omega \|_{\infty}\right.$ is equal to the inverse of the minimum distance from the loop transfer function to the critical point $(-1,0)$ in the Nyquist plot. Robust controller is obtained through minimizing this $H_{\infty}$ norm.

\subsection{Designing approach}

In this section, the design procedure is described. The tuning procedure has two main steps. In the first step, two new design parameters are defined and these parameters are related to the controller parameters. In the next step the optimal values of these two parameters are obtained. Consider two types of processes, stable processes and integrating processes.

\subsubsection{Stable processes}

First, only closed loop response $\left(y_{C L D}(t)\right)$ on step input disturbance $(\mathrm{d}=1$ and $\mathrm{r}=0)$ is considered.

$$
\frac{Y_{C L D}(s)}{D(s)}=\frac{G_{p}(s)}{1+G_{p}(s) G_{c}(s)}=\frac{k_{p}\left(\frac{e^{-\tau s}}{T s+1}\right)}{1+k_{p} k_{c}\left(\frac{e^{\tau s}}{T s+1}\right)\left(1+\frac{1}{T_{i} s}\right)}
$$

No text of specified style in document..12)

(Error!

Approximate delay term in denominator is

$$
e^{-\tau s} \approx 1-\tau s
$$

(Error! No text of specified

style in document..13)

By this approximation a second order polynomial for closed loop characteristic is achieved 


$$
\frac{Y_{C L D}(s)}{D(s)} \approx \frac{\left(\frac{T_{i}}{k_{c}} s e^{-^{-s}}\right)}{\left(\frac{T T_{i}}{k_{c} k_{p}}-\tau T_{i}\right) s^{2}+\left(\frac{T_{i}}{k_{c} k_{p}}+T_{i}-\tau\right) s+1}
$$

(Error! No

text of specified style in document..14)

Using a second order plus dead time model reference transfer function, with two real poles, yields to good trade off between characteristics of transient response and stability of closed-loop system.

$$
\frac{Y_{C L D}(s)}{D(s)} \approx \frac{\left(\frac{T_{i}}{k_{c}} s e^{-\tau}\right)}{\left(\frac{T T_{i}}{k_{c} k_{p}}-\tau T_{i}\right) s^{2}+\left(\frac{T_{i}}{k_{c} k_{p}}+T_{i}-\tau\right) s+1}=\frac{k_{d} s e^{-\tau}}{\left(T_{1} s+1\right)\left(T_{2} s+1\right)}
$$

of specified style in document...15)

(Error! No text

Consider two new design parameters $\alpha$ and $T_{c}$ as

$\alpha=\frac{T_{2}}{T_{1}}>1$ and $T_{c}=T_{1}$

Parameters $k_{c}$ and $T_{i}$ are easily calculated as a function of parameters of the plant model and are given by [43] $k_{c}=f_{1}\left(k_{p}, T, \tau, T_{c}, \alpha\right)=\frac{T\left[(\alpha+1) T_{c}+\tau\right]-\alpha T_{c}^{2}}{k_{p}\left((\alpha+1) \tau T_{c}+\tau^{2}+\alpha T_{c}^{2}\right)}$

of specified style in document..16)

(Error! No text

$$
T_{i}=f_{2}\left(k_{p}, T, \tau, T_{c}, \alpha\right)=\frac{T\left[(\alpha+1) T_{c}+\tau\right]-\alpha T_{c}^{2}}{(T+\tau)}
$$

(Error! No text

of specified style in document..17)

To find the parameter b, closed loop response, ${ }^{y_{C L R}}(t)$ on step input set point $(\mathrm{r}=1)$ and $(\mathrm{d}=0)$ is considered.

The transfer function

$$
\begin{aligned}
& \begin{array}{l}
\frac{Y_{C L R}(s)}{R(s)}=\frac{G_{f f}(s) G_{p}(s)}{1+G_{C}(s) G_{p}(s)}=\frac{k_{c} k_{p}\left(b+\frac{1}{T_{i} s}\right)\left(\frac{e^{-\tau s}}{T s+1}\right)}{1+k_{p} k_{c}\left(\frac{e^{-\tau s}}{T s+1}\right)\left(1+\frac{1}{T_{i} s}\right)} \\
\approx \frac{\left(b T_{i} s+1\right) e^{-\tau s}}{\left(\frac{T T_{i}}{k_{c} k_{p}}-\tau T_{i}\right) s^{2}+\left(\frac{T_{i}}{k_{c} k_{p}}+T_{i}-\tau\right) s+1}=\frac{\left(b T_{i} s+1\right) e^{-\tau s}}{\left(T_{1} s+1\right)\left(T_{2} s+1\right)}
\end{array} \\
& \text { of specified style in document..18) }
\end{aligned}
$$

(Error! No text of specified style in document..19)

To obtain parameter b, it is supposed that pole-zero cancellation is occurred and the slow mode is eliminated. This pole-zero cancellation yields to fast response so the parameter $b$ can be calculated as

$$
b=\frac{\alpha T_{c}}{T_{i}}
$$

\subsubsection{Integrating processes}

Integrating processes can be considered as a typical FOPDT with a very large time constant, The process transfer function is given by 
$G_{p}(s)=\lim _{T \rightarrow \infty} \frac{k_{p} e^{-\tau s}}{T s+1} \cong \frac{k_{p} e^{-\tau s}}{s}$

Therefore, PI tuning formulas for the integrating process are obtained by using (3.11), (3.12) and (3.14) for a large time constant, as follows

$$
K_{c}=\frac{(\alpha+1) T_{c}+\tau}{k_{p}\left((\alpha+1) \tau T_{c}+\tau^{2}+\alpha T_{c}^{2}\right)}
$$

of specified style in document..20)

(Error! No text

$T_{i}=(\alpha+1) T_{c}+\tau$

of specified style in document..21)

(Error! No text

$b=\frac{\alpha T_{c}}{T_{i}}$

of specified style in document..22)

(Error! No text

\subsubsection{Second step}

The main idea that is used here is determination of parameter $\mathrm{b}$. This parameter has direct effect on the resulting controller so it should be chosen such that the closed loop performance is satisfied and the designed controller is physically realizable. To choose parameter $T_{c}$, some important points should be considered:

(i) $T_{c}$ is related to the open loop time constant and delay time. It is true to say that in stable systems $T_{c}$ also should be function of $\frac{\tau}{T}$. And in integrating systems, $T_{c}$ should be function of delay time $\tau$.

(ii) Systems with large delay time should not be expected to have so fast closed loop response in comparison with open loop response. So in this case $T_{c}$ should be a little large.

(iii) For systems with a small value of $\frac{\tau}{T}$ it is possible to choose $T_{c}$ small to have fast response.

(iv) Decreasing $T_{c}$ leads to a fast closed loop response.

\section{Design examples for 2-DOF PI controller structure}

In this different plant processes are first transformed to FOPDT model and then parameters of PI controller are obtained

\subsubsection{Design example 3: Oscillating system}

The system has one real and two complex poles on the LHP of s plane. The real pole contributes in the decay of the response while the imaginary pole contributes for the oscillations in the system. Thus the complex pole will contribute to both decay and oscillations.

The transfer function of the plant is

$$
G(s)=\frac{9}{(s+1)\left(s^{2}+2 s+9\right)}
$$

This system is very common in process control. In order to tune PI parameters using the proposed method, a FOPDT model of the system is required. A simple method based on open loop step response is introduced in [39] which gives following FOPDT model.

$G_{m}(s)=\frac{e^{-0.43 s}}{0.825 s+1}$

The PI controllers are designed using equations (3.20) and (3.21) which give following value of $K_{c}, T_{i}$ and b.

$$
K_{c}=0.64
$$

$T_{i}=1$ 
$b=1.59$

Using these PI controllers, the performances have been studied using simulink block diagram as shown in Figure 4.13

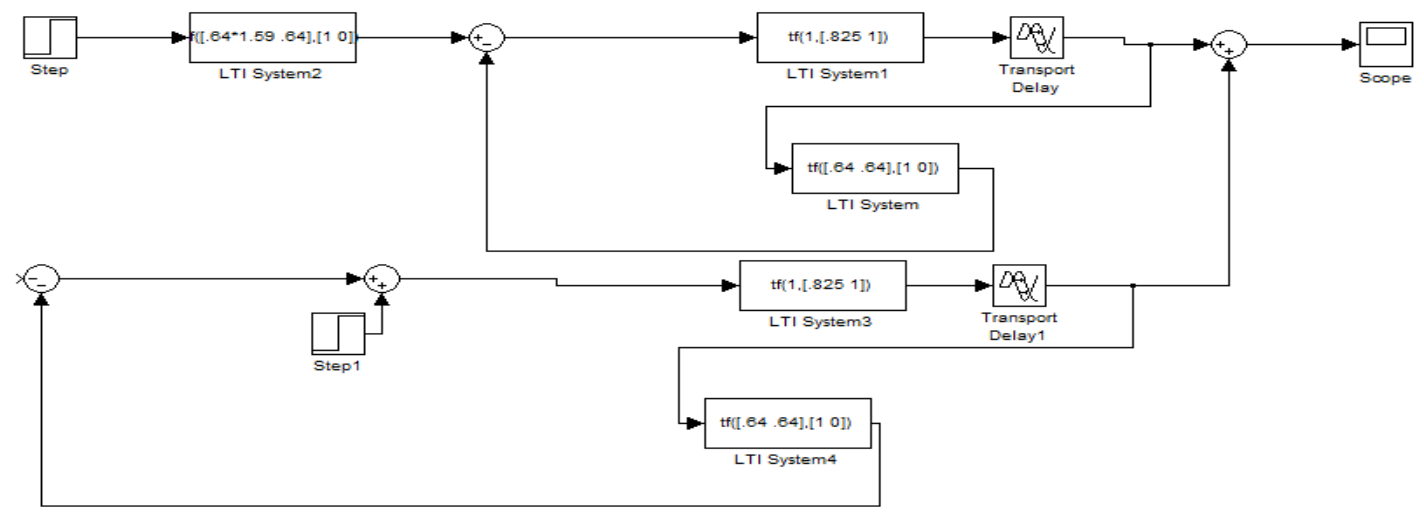

Figure Error! No text of specified style in document..7 Block diagram for the oscillating system The step response of the closed loop system and effect of step disturbance is shown in the Figure 4.14

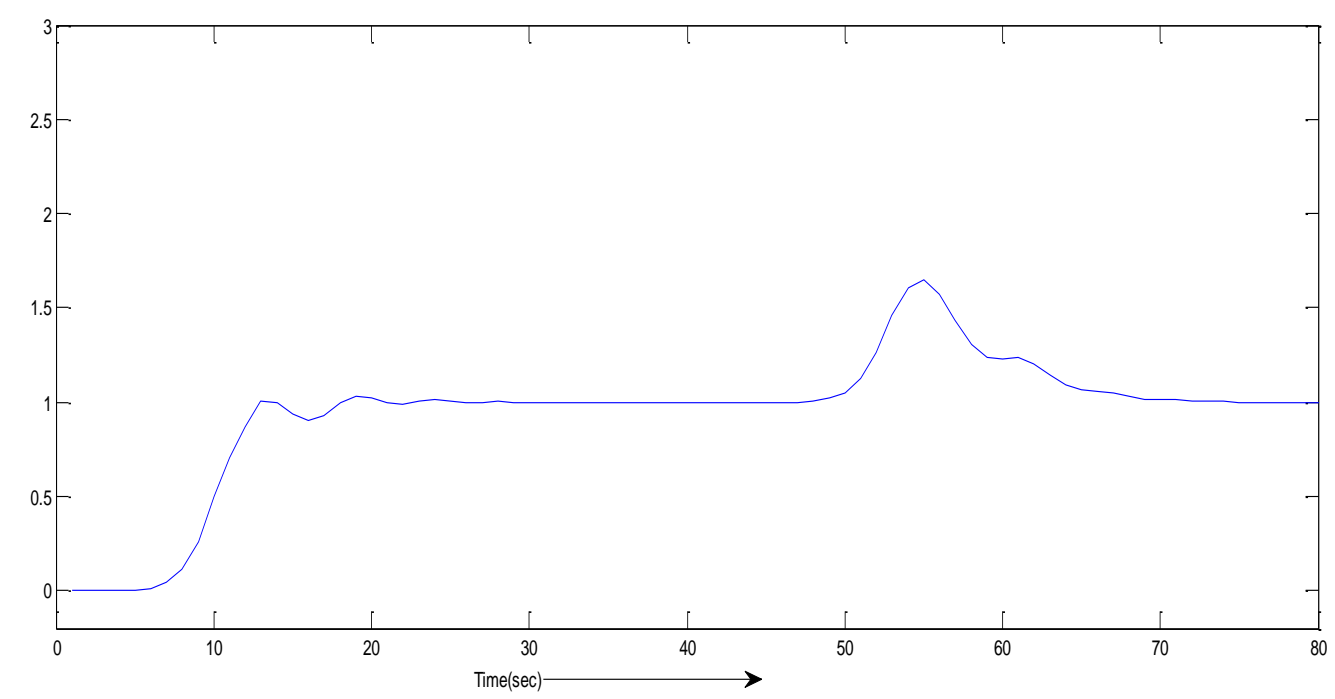

Figure Error! No text of specified style in document..8 Step response for the oscillating system

The step response of the system comes out to satisfactory and it provides excellent disturbance rejection properties which is acting on the system after 50 seconds.

In this chapter, the design approaches for 2-DOF controller structure has been used for controller design taking example 4.1.1.The 2-DOF controller configuration facilitates achievement of time domain and frequency domain responses independently.

\section{Conclusion}

In this work a new tuning approach for 2-DOF PI controller based on FOPDT model of process has been proposed.

The design problem considers three essential requirements of control problem. These requirements are load disturbance rejection, set point regulation and robustness to model uncertainties. The main advantage of proposed method is its simplicity and analytic equations for controller parameters. The simulation results show that, over all, the proposed tuning method can effectively satisfy conflicting design requirements.

\section{Reference}

[1]. J.C. Doyle and G. Stein. "Multivariable feedback design: Concepts for a classical/modern synthesis," IEEE Trans. Automat. Contr. 26(2), 4-16, 1981.

[2]. J. Chen, "Sensitivity integral relations and design trade-offs in linear multivariable feedback systems," IEEE Trans. Automat. Contr 40(10), 1700-1716, 1995. 
[3]. B. A. Francis, A course in $H_{\infty}$ Control theory. Springer-Verlag. Lecture Notes in Control and Information Sciences, 1987.

[4]. M. Green and D. J. N. Limebeer, Linear Robust Control, Prentice Hall Information and System Science Series, 1995.

[5]. D. Youla, H.A. Jabr and J. Bongiorno, "Modern Wiener-Hopf design of optimal controllers, part 2: The multivariable case," IEEE Trans. Automat. Contr. 21(6), 319-338, 1976.

[6]. M. Vidyasagar, Control System Synthesis. 'A factorization approach', MIT Press. Cambridge, Massachusetts, 1985.

[7]. J.C. Doyle, "Synthesis of robust controllers and filters," Proc. of the IEEE Conference on Decision and Control pp. 109-124, 1983.

[8]. C. N. Nett, C. A. Jacobson and N. J. Balas, "A connection between state-space and doubly coprime fractional representations," IEEE Trans. Automat. Contr. 29, 831-832, 1987.

[9]. J.M. Maciejowski, Multivariable Feedback Design. Addison-Wesley Publishing, 1989.

[10]. C.A. Desoer and C.L. Gustafson "Algebraic theory of linear multi variable systems," IEEE Trans. Automat. Contr. 29, 909-917, 1984.

[11]. M. Vidyasagar, H. Schneider and B. A. Francis, "Algebraic and topological aspects of feedback stabilization," IEEE Trans. Automat. Contr. 27(4), 880-894, 1982.

[12]. T. Kailath, Linear Systems, Prentice-Hall, 1980.

[13]. J. Ackerman, Robust Control: Systems with uncertain physical parameters, Apringer Verlag. Communication and Control Engineering Series, 1993.

[14]. K. Zhou, J. C. Doyle and K. Glover, Robust and Optimal Control, Prentice Hall, Inc.. Upper Saddle River, New Jersey, 1995.

[15]. S. P. Boyd, C. H. Barratt, Linear Controller Design. Limits of Performance. system sciences series. Prentice-Hall International, 1991. 